\title{
Well-posed initial-boundary value problem for the harmonic Einstein equations using energy estimates
}

\author{
by \\ H.-O. Kreiss ${ }^{1,2}$, O. Reula ${ }^{3}$, O. Sarbach ${ }^{4}$ and J. Winicour ${ }^{2,5}$ \\ ${ }^{1}$ NADA, Royal Institute of Technology, 10044 Stockholm, Sweden \\ ${ }^{2}$ Albert Einstein Institute, Max Planck Gesellschaft, Am Mühlenberg 1, D-14476 \\ Golm, Germany \\ ${ }^{3}$ FaMAF Universidad Nacional de Cordoba, Cordoba, Argentina 5000 \\ ${ }^{4}$ Instituto de Física y Matemáticas, Universidad Michoacana de San Nicolás de Hi- \\ dalgo, Edificio C-3, C. P. 58040 Morelia, Michoacán, México \\ ${ }^{5}$ Department of Physics and Astronomy, University of Pittsburgh, Pittsburgh, Penn- \\ sylvania 15260, USA
}

\begin{abstract}
In recent work, we used pseudo-differential theory to establish conditions that the initialboundary value problem for second order systems of wave equations be strongly well-posed in a generalized sense. The applications included the harmonic version of the Einstein equations. Here we show that these results can also be obtained via standard energy estimates, thus establishing strong well-posedness of the harmonic Einstein problem in the classical sense.
\end{abstract}

PACS numbers: 04.20.Ex, 04.25.Dm, 04.25.Nx

\section{Introduction}

In the harmonic description of general relativity, the Einstein equations reduce to a constrained system of 10 quasilinear wave equations for the components of the spacetime metric. Recently [1] we used the theory of pseudo-differential operators to prove that one can construct constraint preserving boundary conditions of Sommerfeld type such that the resulting initial-boundary value problem (IBVP) is well-posed in the generalized sense. We show in this paper that the decisive estimate can also be obtained by integration by parts. 
It follows that the full quasilinear system can be treated by standard energy estimates to establish that the harmonic IBVP is strongly well-posed in the classical sense [2].

Our results have broad application to other systems of second order wave equations besides general relativity, e.g. to elasticity theory, acoustics and electromagnetic theory. Most analytic and computational treatments of the IBVP utilize the well-developed theory of first order symmetric hyperbolic systems. We develop our results here in their natural second order form, which avoids the integrability constraints associated with the extra variables introduced in a reduction to first order.

In view of the wide range of potential applications, instead of the geometrical notation of general relativity, we present our main results in Sec's $2-3$ in a style familiar to a broad audience of computational mathematicians and physicists. We use the notation and definitions of the classic treatise [2] on the IBVP. In these sections, we treat systems of wave equations with constant coefficients, as arise in the frozen coefficient form of the harmonic Einstein equations. In Appendix 1, we show that our results extend locally in time to the well-posedness of quasilinear problems, such as the harmonic IBVP.

There is an intimate interplay between the treatment of the analytic theory using energy estimates and its finite difference approximation $[2,3]$. The proof of existence of analytic solutions outlined in Appendix 1 is based upon a convergent finite difference approximation incorporating semi-discrete energy estimates via summation by parts. Conversely, using summation by parts, the energy estimates of the analytic theory can be parroted by discrete energy estimates which guarantee the stability of a finite difference approximation [3]. In previous treatments of the second order wave equation, the discrete energy approach has been used to develop stable difference algorithms for Neumann and Dirichlet boundary conditions $[4,5]$ and this treatment has been extended to the Einstein equations [6]. The results presented here provide a guide for applying the discrete energy approach to second order systems with a wide range of boundary conditions. These include the Sommerfeld condition, which has important application to outgoing wave problems, but also more complicated conditions involving derivatives tangential to the boundary.

The set of constraint-preserving boundary conditions allowed for the Einstein system is quite extensive. The selection of a "preferred" choice, e.g. Dirichlet, Neumann or Sommerfeld, rests upon additional geometrical or physical criteria depending upon the nature of the problem. An important example is the description of an isolated radiating system, for which the physically appropriate boundary condition should be adapted to the absence 
of external influences. Unless the treatment of such a system is extended to infinity, this requires introduction of an artificial boundary for which boundary data may not be known, so that it becomes advantageous to use a boundary condition for which homogeneous data is a good approximation. We defer this issue to future work where we will present a geometric version of the results of this paper to formulate boundary conditions appropriate for an isolated gravitational system. Additional subtleties arise in the treatment of boundaries which are moving, such as an oscillating conducting boundary in an electromagnetic wave problem or the artificial boundaries that arise in the dynamically curved spacetime of general relativity. In Appendix 2, we illustrate for the wave equation on a curved space background how the geometric approach can be used to simplify the formulation of energy estimates for such moving boundaries.

\section{The main estimate}

Consider the wave equation

$$
u_{t t}=u_{x x}+u_{y y}+u_{z z}+F
$$

on the half-space

$$
x \geq 0, \quad-\infty<y<\infty, \quad-\infty<z<\infty
$$

with boundary conditions

$$
\alpha u_{t}=u_{x}+\beta_{1} u_{y}+\beta_{2} u_{z}+\alpha q, \quad \text { for } \quad x=0, \alpha>0, \beta_{1}^{2}+\beta_{2}^{2}<\alpha^{2},
$$

with boundary data $q$ and initial data

$$
u=f_{1}, \quad u_{t}=f_{2}, \quad t=0
$$

of compact support. The subscripts $(t, x, y, z)$ denote partial derivatives, e.g $u_{t}=\frac{\partial u}{\partial t}$. We assume that all coefficients, the data and the solution are real and that $\alpha>0, \beta_{j}$ are constants. Also, we use the notation

$$
(u, v), \quad\|u\|^{2}=(u, u) ; \quad(u, v)_{B}, \quad\|u\|_{B}^{2}=(u, u)_{B}
$$

to denote the $L_{2}$-scalar product and norm over the half-space and boundary space, respectively. 
Strong well-posedness of an IBVP extends the requirements of a well-posed Cauchy problem to include estimates of boundary values [2]. In order to adapt the standard definition to second order systems, we write $\mathbf{u}=\left(u, u_{t}, u_{x}, u_{y}, u_{z}\right)$ to represent the solution $u$ and its derivatives; and similarly for the initial data we write $\mathbf{f}_{1}=\left(f_{1}, f_{1 x}, f_{1 y}, f_{1 z}\right)$. For the problem (1)-(3), strong well-posedness requires the existence of a solution satisfying the estimate

$$
\|\mathbf{u}(\mathbf{t})\|^{2}+\int_{0}^{t}\|\mathbf{u}(\mathbf{s})\|_{B}^{2} d s \leq K_{T}\left(\left\|\mathbf{f}_{1}\right\|^{2}+\left\|f_{2}\right\|^{2}+\int_{0}^{t}\|F(s)\|^{2} d s+\int_{0}^{t}\|q(s)\|_{B}^{2} d s\right)
$$

where for every finite time interval $0 \leq t \leq T$ the constant $K_{T}$ is independent of $F, f_{1}, f_{2}$ and $q$.

In [1] we used pseudo-differential theory to prove that the problem (1)-(3) is wellposed in the generalized sense and that it is boundary stable. The proof in [1] is only given in $2 \mathrm{D}$. In $3 \mathrm{D}$ the necessary and sufficient condition for $\alpha$ is $\alpha>\sqrt{\beta_{1}^{2}+\beta_{2}^{2}}$. This same inequality governs the strong well-posedness of the problem. (It corresponds to the timelike property of the vector field $T^{b}$ in the geometric treatment of Appendix 2.)

We now want to prove that results can also be obtained in terms of standard estimates using integration by parts. For simplicity of presentation, we restrict our attention here to estimates of the derivatives of $u$. An estimate for $u$ itself can easily be obtained by the change of variable $u \rightarrow e^{\gamma t} u$, as described in Appendix 1. We start with

Lemma 1. Let $\gamma_{1}, \gamma_{2}$ be real and $\gamma_{1}^{2}+\gamma_{2}^{2}<1$. Then

$$
E=\left\|u_{t}\right\|^{2}+\left\|u_{x}\right\|^{2}+\left\|u_{y}\right\|^{2}+\left\|u_{z}\right\|^{2}-2\left(u_{t}, \gamma_{1} u_{y}+\gamma_{2} u_{z}\right)
$$

is a norm for the derivatives $\left(u_{t}, u_{x}, u_{y}, u_{z}\right)$.

Proof. Since $\gamma_{1}^{2}+\gamma_{2}^{2}<1$, we can choose $\delta$ with $0<\delta<1$ such that

$$
\begin{aligned}
2\left|\left(u_{t}, \gamma_{1} u_{y}+\gamma_{2} u_{z}\right)\right| & \leq(1-\delta)\left\|u_{t}\right\|^{2}+\frac{1}{1-\delta}\left(\gamma_{1}^{2}+\gamma_{2}^{2}\right)\left(\left\|u_{y}\right\|^{2}+\left\|u_{z}\right\|^{2}\right) \\
& \leq(1-\delta)\left(\left\|u_{t}\right\|^{2}+\left\|u_{y}\right\|^{2}+\left\|u_{z}\right\|^{2}\right)
\end{aligned}
$$

This proves the lemma.

Let $\gamma_{1}=\frac{\beta_{1}}{\alpha}, \gamma_{2}=\frac{\beta_{2}}{\alpha}$. Now we can prove that there is a standard energy estimate. 
Theorem 1. The solution of (1)-(3) satisfies the energy estimate

$$
\frac{\partial}{\partial t} E+\frac{1}{\alpha}\left\|u_{x}\right\|_{B}^{2} \leq E+\|F\|^{2}+\alpha\|q\|_{B}^{2}
$$

Proof. Integration by parts gives us

$$
\begin{aligned}
\frac{\partial}{\partial t}\left\|u_{t}\right\|^{2}=2\left(u_{t}, u_{t t}\right)=-\frac{\partial}{\partial t} & \left(\left\|u_{x}\right\|^{2}+\left\|u_{y}\right\|^{2}+\left\|u_{z}\right\|^{2}\right)+2\left(u_{t}, F\right)-2\left(u_{t}, u_{x}\right)_{B} \\
2 \frac{\partial}{\partial t}\left(u_{t}, \gamma_{1} u_{y}+\gamma_{2} u_{z}\right) & =2\left(u_{t t}, \gamma_{1} u_{y}+\gamma_{2} u_{z}\right) \\
& =-2\left(u_{x}, \gamma_{1} u_{y}+\gamma_{2} u_{z}\right)_{B}+2\left(F, \gamma_{1} u_{y}+\gamma_{2} u_{z}\right),
\end{aligned}
$$

where, for example, we have used $\left(u_{y y}, u_{z}\right)=-\left(u_{y}, u_{y z}\right)=0$. Since (2) implies

$$
2\left(u_{t}, u_{x}\right)_{B}=\frac{2}{\alpha}\left\|u_{x}\right\|_{B}^{2}+2\left(u_{x}, \gamma_{1} u_{y}+\gamma_{2} u_{z}\right)_{B}+2\left(u_{x}, q\right)_{B}
$$

by subtracting (7) from (6) we obtain

$$
\begin{aligned}
\frac{\partial}{\partial t} E & =2\left(u_{t}-\gamma_{1} u_{y}-\gamma_{2} u_{z}, F\right)-\frac{2}{\alpha}\left\|u_{x}\right\|_{B}^{2}-2\left(u_{x}, q\right)_{B} \\
& \leq\left\|u_{t}-\gamma_{1} u_{y}-\gamma_{2} u_{z}\right\|^{2}+\|F\|^{2}-\frac{1}{\alpha}\left\|u_{x}\right\|_{B}^{2}+\alpha\|q\|_{B}^{2}
\end{aligned}
$$

The identity

$$
\left\|u_{t}-\gamma_{1} u_{y}-\gamma_{2} u_{z}\right\|^{2}=E-\left\|u_{x}\right\|^{2}-\left\|u_{y}\right\|^{2}-\left\|u_{z}\right\|^{2}+\left\|\gamma_{1} u_{y}+\gamma_{2} u_{z}\right\|^{2}
$$

then implies (5) and thus proves the theorem.

The theorem tells us that we can estimate

$$
E(T) \text { and } \int_{0}^{T}\left\|u_{x}\right\|_{B}^{2} d t \text { in terms of } E(0), \int_{0}^{T}\|F\|^{2} d t \text { and } \int_{0}^{T}\|q\|_{B}^{2} d t \text {. }
$$

For the application to the Einstein equations we also need estimates of the boundary norms of $u_{y}$ and $u_{z}$. We have

$$
\begin{aligned}
\frac{\partial}{\partial t}\left(u_{x}, u_{t}\right) & =\left(u_{x t}, u_{t}\right)+\left(u_{x}, u_{t t}\right) \\
& =-\frac{1}{2}\left\|u_{t}\right\|_{B}^{2}+\left(u_{x}, u_{x x}\right)+\left(u_{x}, u_{y y}\right)+\left(u_{x}, u_{z z}\right)+\left(u_{x}, F\right) \\
& =-\frac{1}{2}\left\|u_{t}\right\|_{B}^{2}-\frac{1}{2}\left\|u_{x}\right\|_{B}^{2}+\frac{1}{2}\left\|u_{y}\right\|_{B}^{2}+\frac{1}{2}\left\|u_{z}\right\|_{B}^{2}+\left(u_{x}, F\right) .
\end{aligned}
$$


The boundary conditions (2) give us, for any $\delta$ with $0<\delta<1$,

$$
\begin{aligned}
\left\|u_{t}\right\|_{B}^{2} & =\left\|\gamma_{1} u_{y}+\gamma_{2} u_{z}+\frac{1}{\alpha} u_{x}+q\right\|_{B}^{2} \\
& \leq\left\|\gamma_{1} u_{y}+\gamma_{2} u_{z}\right\|_{B}^{2}+2\left\|\gamma_{1} u_{y}+\gamma_{2} u_{z}\right\|_{B}\left\|\frac{1}{\alpha} u_{x}+q\right\|_{B}+\left\|\frac{1}{\alpha} u_{x}+q\right\|_{B}^{2} \\
& \leq(1+\delta)\left\|\gamma_{1} u_{y}+\gamma_{2} u_{z}\right\|_{B}^{2}+\left(1+\frac{1}{\delta}\right)\left\|\frac{1}{\alpha} u_{x}+q\right\|_{B}^{2} \\
& \leq(1+\delta)\left(\gamma_{1}^{2}+\gamma_{2}^{2}\right)\left(\left\|u_{y}\right\|_{B}^{2}+\left\|u_{z}\right\|_{B}^{2}\right)+\left(1+\frac{1}{\delta}\right)\left\|\frac{1}{\alpha} u_{x}+q\right\|_{B}^{2} .
\end{aligned}
$$

Since $\varrho:=\gamma_{1}^{2}+\gamma_{2}^{2}<1$ we can choose $\delta$ such that $(1+\delta) \varrho \leq(1-\delta)$. Therefore, by (8),

$$
\delta\left(\left\|u_{y}\right\|_{B}^{2}+\left\|u_{z}\right\|_{B}^{2}\right) \leq\left(1+\frac{1}{\delta}\right)\left\|\frac{1}{\alpha} u_{x}+q\right\|_{B}^{2}+\left\|u_{x}\right\|_{B}^{2}+2 \frac{\partial}{\partial t}\left(u_{x}, u_{t}\right)-2\left(u_{x}, F\right) .
$$

Since $\left(u_{x}, u_{t}\right)$ can be estimated by $E$, we have proved

\section{Theorem 2.}

$$
\begin{aligned}
& \int_{0}^{T}\left(\left\|u_{t}\right\|_{B}^{2}+\left\|u_{x}\right\|_{B}^{2}+\left\|u_{y}\right\|_{B}^{2}+\left\|u_{z}\right\|_{B}^{2}\right) d t \\
& \quad \leq \text { const. }\left(E(0)+\int_{0}^{T}\|F\|^{2} d t+\int_{0}^{T}\|q\|_{B}^{2} d t\right) .
\end{aligned}
$$

The results can easily be generalized to half-plane problems for wave equations of the general form

$$
u_{t t}=P_{0} u_{t}+P_{1} u, \quad x_{1} \geq 0, \quad-\infty<x_{j}<\infty, j=2,3
$$

with boundary conditions

$$
\alpha u_{t}=u_{x_{1}}+\beta_{1} u_{x_{2}}+\beta_{2} u_{x_{3}}, \quad x_{1}=0
$$

Here

$$
P_{0}=\sum_{j=1}^{3} c_{j} \partial / \partial x_{j}, \quad P_{1}=\sum_{i, j=1}^{3} a_{i j} \partial^{2} / \partial x_{i} \partial x_{j}, a_{i i}>0
$$

and $P_{1}$ is strongly elliptic.

By general coordinate transformations, we can transform $(9),(10)$ to the simple problem (1)-(3), except if the coordinate system moves normal to the boundary. To discuss this case we consider a boundary moving with constant velocity $c / \sqrt{1+c^{2}}$ in the $x$-direction and transform (1) according to

$$
x=\frac{x^{\prime}+c t^{\prime}}{\sqrt{1+c^{2}}}, \quad t=t^{\prime}, \quad y=y^{\prime}, \quad z=z^{\prime},
$$


so that the moving boundary is located at $x^{\prime}=0$. After dropping the primes, we obtain

$$
u_{t t}=2 c u_{x t}+u_{x x}+u_{y y}+u_{z z}+F
$$

and we consider the problem $\left(1^{\prime}\right),(2),(3)$. The same pseudo-differential technique as in Section 2 of [1] shows that this new problem is well-posed in the generalized sense if and only if

$$
\frac{\alpha+c}{\sqrt{1+c^{2}}}>\sqrt{\beta_{1}^{2}+\beta_{2}^{2}}
$$

Subject to this inequality, in Appendix 2 we use a geometric approach to establish strong well-posedness for this moving boundary problem by the energy method.

\section{The Einstein equations}

We consider the half-plane problem treated in [1] for the harmonic Einstein equations, which we know describe. In the frozen coefficient formalism based upon an orthonormal frame, the components of the densitized spacetime metric satisfy the wave equations

$$
\begin{gathered}
\left(-\frac{\partial^{2}}{\partial t^{2}}+\frac{\partial^{2}}{\partial x^{2}}+\frac{\partial^{2}}{\partial y^{2}}+\frac{\partial^{2}}{\partial z^{2}}\right)\left(\begin{array}{cccc}
\gamma^{t t} & \gamma^{t x} & \gamma^{t y} & \gamma^{t z} \\
\gamma^{t x} & \gamma^{x x} & \gamma^{x y} & \gamma^{x z} \\
\gamma^{t y} & \gamma^{x y} & \gamma^{y y} & \gamma^{y z} \\
\gamma^{t z} & \gamma^{x z} & \gamma^{y z} & \gamma^{z z}
\end{array}\right)=F \\
x \geq 0, t \geq 0,-\infty<y<\infty,-\infty<z<\infty
\end{gathered}
$$

where $F$ consists of lower order terms.

These equations are subject to the constraints

$$
\begin{aligned}
C^{t} & =\frac{\partial}{\partial t} \gamma^{t t}+\frac{\partial}{\partial x} \gamma^{t x}+\frac{\partial}{\partial y} \gamma^{t y}+\frac{\partial}{\partial z} \gamma^{t z}=0, \\
C^{x} & =\frac{\partial}{\partial t} \gamma^{t x}+\frac{\partial}{\partial x} \gamma^{x x}+\frac{\partial}{\partial y} \gamma^{x y}+\frac{\partial}{\partial z} \gamma^{x z}=0, \\
C^{y} & =\frac{\partial}{\partial t} \gamma^{t y}+\frac{\partial}{\partial x} \gamma^{x y}+\frac{\partial}{\partial y} \gamma^{y y}+\frac{\partial}{\partial z} \gamma^{y z}=0, \\
C^{z} & =\frac{\partial}{\partial t} \gamma^{t z}+\frac{\partial}{\partial x} \gamma^{x z}+\frac{\partial}{\partial y} \gamma^{y z}+\frac{\partial}{\partial z} \gamma^{z z}=0 .
\end{aligned}
$$

Because the constraints satisfy homogeneous wave equations, if $C^{a}(0, x, y, z)=0$ and $\frac{\partial}{\partial t} C^{a}(0, x, y, z)=0, a=(t, x, y, z)$, then they remain zero at later times if $C^{a}=0$ are part of the boundary conditions for (11) at $x=0$. 
As in [1], we consider the choice of boundary conditions

$$
\begin{aligned}
& \frac{\partial}{\partial t}\left(\begin{array}{c}
\gamma^{t t} \\
\gamma^{t x} \\
\gamma^{x x} \\
\gamma^{t y} \\
\gamma^{x y} \\
\gamma^{t z} \\
\gamma^{x z} \\
\gamma^{y y} \\
\gamma^{y z} \\
\gamma^{z z}
\end{array}\right)+\frac{\partial}{\partial x}\left(\begin{array}{cccccccccc}
0 & 1 & 0 & 0 & 0 & 0 & 0 & 0 & 0 & 0 \\
0 & 0 & 1 & 0 & 0 & 0 & 0 & 0 & 0 & 0 \\
a_{1} & a_{2} & a_{3} & 0 & 0 & 0 & 0 & 0 & 0 & 0 \\
0 & 0 & 0 & 0 & 1 & 0 & 0 & 0 & 0 & 0 \\
0 & 0 & 0 & b_{1} & b_{2} & 0 & 0 & 0 & 0 & 0 \\
0 & 0 & 0 & 0 & 0 & 0 & 1 & 0 & 0 & 0 \\
0 & 0 & 0 & 0 & 0 & c_{1} & c_{2} & 0 & 0 & 0 \\
0 & 0 & 0 & 0 & 0 & 0 & 0 & -1 & 0 & 0 \\
0 & 0 & 0 & 0 & 0 & 0 & 0 & 0 & -1 & 0 \\
0 & 0 & 0 & 0 & 0 & 0 & 0 & 0 & 0 & -1
\end{array}\right)\left(\begin{array}{c}
\gamma^{t t} \\
\gamma^{t x} \\
\gamma^{x x} \\
\gamma^{t y} \\
\gamma^{x y} \\
\gamma^{t z} \\
\gamma^{x z} \\
\gamma^{y y} \\
\gamma^{y z} \\
\gamma^{z z}
\end{array}\right) \\
& +\frac{\partial}{\partial y}\left(\begin{array}{cccccccccc}
0 & 0 & 0 & 1 & 0 & 0 & 0 & 0 & 0 & 0 \\
0 & 0 & 0 & 0 & 1 & 0 & 0 & 0 & 0 & 0 \\
0 & 0 & 0 & 0 & 0 & 0 & 0 & 0 & 0 & 0 \\
0 & 0 & 0 & 0 & 0 & 0 & 0 & 1 & 0 & 0 \\
0 & 0 & 0 & 0 & 0 & 0 & 0 & 0 & 0 & 0 \\
0 & 0 & 0 & 0 & 0 & 0 & 0 & 0 & 1 & 0 \\
0 & 0 & 0 & 0 & 0 & 0 & 0 & 0 & 0 & 0 \\
0 & 0 & 0 & 0 & 0 & 0 & 0 & 0 & 0 & 0 \\
0 & 0 & 0 & 0 & 0 & 0 & 0 & 0 & 0 & 0 \\
0 & 0 & 0 & 0 & 0 & 0 & 0 & 0 & 0 & 0
\end{array}\right)\left(\begin{array}{c}
\gamma^{t t} \\
\gamma^{t x} \\
\gamma^{x x} \\
\gamma^{t y} \\
\gamma^{x y} \\
\gamma^{t z} \\
\gamma^{x z} \\
\gamma^{y y} \\
\gamma^{y z} \\
\gamma^{z z}
\end{array}\right) \\
& +\frac{\partial}{\partial z}\left(\begin{array}{cccccccccc}
0 & 0 & 0 & 0 & 0 & 1 & 0 & 0 & 0 & 0 \\
0 & 0 & 0 & 0 & 0 & 0 & 1 & 0 & 0 & 0 \\
0 & 0 & 0 & 0 & 0 & 0 & 0 & 0 & 0 & 0 \\
0 & 0 & 0 & 0 & 0 & 0 & 0 & 0 & 1 & 0 \\
0 & 0 & 0 & 0 & 0 & 0 & 0 & 0 & 0 & 0 \\
0 & 0 & 0 & 0 & 0 & 0 & 0 & 0 & 0 & 1 \\
0 & 0 & 0 & 0 & 0 & 0 & 0 & 0 & 0 & 0 \\
0 & 0 & 0 & 0 & 0 & 0 & 0 & 0 & 0 & 0 \\
0 & 0 & 0 & 0 & 0 & 0 & 0 & 0 & 0 & 0 \\
0 & 0 & 0 & 0 & 0 & 0 & 0 & 0 & 0 & 0
\end{array}\right)\left(\begin{array}{c}
\gamma^{t t} \\
\gamma^{t x} \\
\gamma^{x x} \\
\gamma^{t y} \\
\gamma^{x y} \\
\gamma^{t z} \\
\gamma^{x z} \\
\gamma^{y y} \\
\gamma^{y z} \\
\gamma^{z z}
\end{array}\right)=q
\end{aligned}
$$

where $q$ represents the boundary data. Here $a_{1}, a_{2}, a_{3}, b_{1}, b_{2}$ and $c_{1}, c_{2}$ are real constants such that the eigenvalues $\lambda_{j}$ of

$$
\left(\begin{array}{ccc}
0 & 1 & 0 \\
0 & 0 & 1 \\
a_{1} & a_{2} & a_{3}
\end{array}\right), \quad\left(\begin{array}{cc}
0 & 1 \\
b_{1} & b_{2}
\end{array}\right) \quad \text { and } \quad\left(\begin{array}{cc}
0 & 1 \\
c_{1} & c_{2}
\end{array}\right)
$$

are real and negative. The constraints vanish on the boundary provided $q^{t a}=0, a=$ $(t, x, y, z)$.

We can now obtain standard energy estimates for the Einstein equations both in the interior and on the boundary. The following theorem strengthens the results in Section 3 of [1] to strong well-posedness: 
Theorem 3. The half-plane problem for the system (11) with constraints (12) and boundary conditions (13) is strongly well-posed if the eigenvalues of the matrices (14) are real and negative.

Proof. As explained in Appendix 1, it suffices to show that all the first derivatives are bounded. We start with the last components. Since the boundary conditions do not contain terms in the tangential direction, Theorems 1 and 2 tell us that all the first derivatives of $\gamma^{z z}$ can be bounded in terms of $\|F\|$ and $\|q\|_{B}$. Thus we gain one derivative both on the boundary and in the interior. The same result holds for the derivatives of $\gamma^{y z}$ and $\gamma^{y y}$.

In the same way as in [1], the boundary conditions for $\gamma^{t z}, \gamma^{x z}$ can be decoupled by a unitary matrix $U$ such that

$$
U\left(\begin{array}{cc}
0 & 1 \\
c_{1} & c_{2}
\end{array}\right) U^{*}=-\left(\begin{array}{cc}
\lambda_{1} & c_{12} \\
0 & \lambda_{2}
\end{array}\right), \quad \lambda_{1}, \lambda_{2}>0
$$

Introducing new variables by

$$
\left(\begin{array}{c}
\tilde{\gamma}^{t z} \\
\tilde{\gamma}^{x z}
\end{array}\right)=U\left(\begin{array}{c}
\gamma^{t z} \\
\gamma^{x z}
\end{array}\right)
$$

we obtain the equations

$$
\frac{\partial}{\partial t}\left(\begin{array}{c}
\tilde{\gamma}^{t z} \\
\tilde{\gamma}^{x z}
\end{array}\right)=\left(\begin{array}{cc}
\lambda_{1} & c_{12} \\
0 & \lambda_{2}
\end{array}\right) \frac{\partial}{\partial x}\left(\begin{array}{c}
\tilde{\gamma}^{t z} \\
\tilde{\gamma}^{x z}
\end{array}\right)+\left(\begin{array}{c}
\tilde{q}^{t z} \\
\tilde{q}^{x z}
\end{array}\right)
$$

where

$$
\left(\begin{array}{c}
\tilde{q}^{t z} \\
\tilde{q}^{x z}
\end{array}\right)=U\left(-\frac{\partial}{\partial y}\left(\begin{array}{c}
\gamma^{y z} \\
0
\end{array}\right)-\frac{\partial}{\partial z}\left(\begin{array}{c}
\gamma^{z z} \\
0
\end{array}\right)+\left(\begin{array}{c}
q^{t z} \\
q^{x z}
\end{array}\right)\right) .
$$

We have already estimates of $\tilde{q}^{t z}$ and $\tilde{q}^{x z}$. Therefore we can estimate all the first derivatives of $\tilde{\gamma}^{x z}$ in terms of $\|F\|$ and $\|q\|_{B}$. The same is true for $\tilde{\gamma}^{t z}$. This process can be continued for the remaining components. Thus we have proved Theorem 3 of [1], where we can remove "in the generalized sense".

Theorem 3 is also valid when the matrices of (13) for the tangential derivatives are upper triangular, i.e. only terms above the diagonal are not zero (or equivalent to that form by unitary transformation). This allows the sequential argument in the proof. It also generalizes to full matrices which are sufficiently close to upper triangular form. A fuller discussion of the most general case will be given in future work.

Local existence theorems for quasilinear equations follow by iteration of the linearized equations, as described in Appendix 1. These results establish strong well-posedness, locally in time, of the quasilinear harmonic IBVP. 


\section{Discussion}

We have shown that the results of [1], obtained using pseudo-differential theory, can also be derived in a more transparent way based upon integration by parts to establish strong well-posedness of a broad class of IBVP's governed by second order quasilinear wave equations. The underlying arguments require no need to rewrite the wave equations as a first order system. As shown in Appendix 1, for smooth data there exist estimates for arbitrarily high derivatives (which is a key requirement for treating the quasilinear case). The boundary conditions are flexible and are stable against perturbation of their coefficients. These properties are important for numerical calculations.

We anticipate that these results will have application to the broad class of problems based upon second order wave equations. For the standard wave equation (1), the chief restriction is that the coefficient $\alpha$ of $u_{t}$ in the boundary condition (2) is positive and satisfies $\alpha^{2}>\beta_{1}^{2}+\beta_{2}^{2}$; for the general wave equation on a curved space background, the corresponding restriction is that the vector $T^{a}$ introduced in Appendix 2 is timelike and future directed. This encompasses boundary conditions of Sommerfeld type. The application to Maxwell's equations expressed in terms of a vector potential in the Lorentz gauge is straightforward.

A prime motivation for this work is the formulation of a well-posed IBVP for the system of harmonic Einstein equations. The importance of numerical simulations in general relativity has spurred a large number of works which have established many of the necessary ingredients for a well-posed IBVP. For a review see [7]. The first complete well-posed formulation was given by Friedrich and Nagy [8] for a version of Einstein's equations in which the curvature tensor, i.e. quantities constructed out of second derivatives of the metric, was included in the evolved variables. Choquet-Bruhat's first proof of the well-posedness of the Cauchy problem for Einstein's equation was given in the harmonic formulation [9]. It is satisfying to extend her work to the harmonic IBVP. Our results also immediately apply to the generalized harmonic formulation in which harmonic forcing terms are allowed [10], since this does not change the principle part of the system.

Since the pioneering results of Pretorius [11,12], there has been rapid progress in the development of numerical codes based upon the generalized harmonic formulation with the capabilty of simulating relativistic binaries consisting of black holes or relativistic stars [13-15]. This difficult problem requires extensive computational tools that do not enter the analytic treatment considered here, namely grid refinement and numerical dissipation. 
Nevertheless, our results provide the analytic background necessary to justify a successful numerical treatment and, at least for finite difference codes, they provide further guidance on how to establish robust boundary conditions. Examples of the boundary conditions considered here have been incorporated in a unigrid, second differential order, harmonic code and successfully tested on model problems [16]. It is beyond the scope of the present work to suggest how they might be incorporated in specific black hole codes. However, in future work we will show how the geometric approach of Appendix 2 can be further developed to yield boundary conditions which are well tailored to the treatment of isolated astrophysical systems.

\section{Appendix 1. The quasilinear case}

The preceding energy estimates establish that a solution of the IBVP with frozen coefficients is unique and depends continuously on the data. In this section we want to show that local existence theorems and energy estimates for second order quasilinear wave equations are proved in the same way as for first order symmetric hyperbolic systems. It all depends on a priori estimates for arbitrarily high derivatives of the solutions of linear equations with variable coefficients. Consider the halfplane problem for

$$
u_{t t}=P u+R u+F, \quad x \geq 0, \quad-\infty<y<\infty,
$$

with boundary conditions

$$
\alpha u_{t}=u_{x}-\gamma u+r u+q, \quad \alpha(y, z, t) \geq \delta>0
$$

and initial data

$$
u(t=0)=f_{1}, \quad u_{t}(t=0)=f_{2}
$$

Here

$$
P u=\left(a u_{x}\right)_{x}+\left(b u_{y}\right)_{y}-2 \gamma u_{t}-\gamma^{2} u
$$

and

$$
R u=c_{1} u_{t}+c_{2} u_{x}+c_{3} u_{y}+c_{4} u .
$$

$R u$ are terms of lower (first and zeroth) differential order. All coefficients are smooth functions of $x, y, t$ and $a \geq a_{0}>0, b \geq b_{0}>0, a_{0}, b_{0}$ and $\delta$ are strictly positive constants. The initial data are smooth functions which are compatible with the boundary conditions. Here $\gamma>0$ is a constant obtained by the change of variables $u \rightarrow e^{\gamma t} u^{\prime}$ and then deleting the 'prime'. This introduces the term $\gamma^{2}\|u\|^{2}$ in the energy $E$ in (18), which provides an estimate of $\|u\|^{2}$. 
Lemma 2. There is an energy estimate which is stable against lower order perturbations.

Proof. Integration by parts gives

$$
\begin{aligned}
\frac{\partial}{\partial t} E & :=\frac{\partial}{\partial t}\left(\left\|u_{t}\right\|^{2}+\left(u_{x}, a u_{x}\right)+\left(u_{y}, b u_{y}\right)+\gamma^{2}\|u\|^{2}\right) \\
& =-4 \gamma\left\|u_{t}\right\|^{2}+2\left(u_{t}, F\right)+2\left(u_{t}, R u\right)-2\left(u_{t}, a u_{x}\right)_{B} \\
& +a_{t}\left\|u_{x}\right\|^{2}+b_{t}\left\|u_{y}\right\|^{2} \\
& \leq \text { const. }\left(\|F\|^{2}+E\right)-2\left(u_{t}, a u_{x}\right)_{B} .
\end{aligned}
$$

Using the boundary conditions gives

$$
\begin{aligned}
-\left(u_{t}, a u_{x}\right)_{B} & =-\left(u_{t}, \alpha a u_{t}\right)_{B}-\left(u_{t}, \gamma a u\right)_{B}+\left(u_{t}, r u+q\right)_{B} \\
& =-\left(u_{t}, \alpha a u_{t}\right)_{B}-\left(u_{t}, \gamma a_{0} u\right)_{B}-\left(u_{t}, \gamma\left(a-a_{0}\right) u\right)_{B}+\left(u_{t}, a r u+a q\right)_{B} \\
& \leq-\frac{1}{2} \gamma a_{0} \frac{\partial}{\partial t}\|u\|_{B}^{2}-\frac{1}{2}\left(u_{t}, \alpha a u_{t}\right)_{B}+\text { const. }\left(\|u\|_{B}^{2}+\|q\|_{B}^{2}\right) .
\end{aligned}
$$

Therefore, we obtain from (18)

$$
\begin{aligned}
& \frac{\partial}{\partial t}\left(E+\gamma a_{0}\|u\|_{B}^{2}+\left(u_{t}, \alpha a u_{t}\right)_{B}\right) \\
& \leq \text { const. }\left(E+\|u\|_{B}^{2}+\|F\|^{2}+\|q\|_{B}^{2}\right) .
\end{aligned}
$$

This proves the lemma.

Now we can estimate the derivatives. Let $v=u_{y}, w=u_{t}$. Differentiating the differential equation gives us

$$
\begin{gathered}
v_{t t}=P v+R v+R_{y} u+\left(a_{y} u_{x}\right)_{x}+\left(b_{y} v\right)_{y}+F_{y}, \\
w_{t t}=P w+R w+R_{t} u+\left(a_{t} u_{x}\right)_{x}+\left(b_{t} v\right)_{y}+F_{t},
\end{gathered}
$$

respectively.

$R_{y} u$ and $R_{t} u$ are linear combinations of first derivatives of $u$ which we have already estimated and can be considered part of the forcing.

The differential equation (15) tells us that

$$
a u_{x x}=w_{t}-b v_{y}+\text { terms we have already estimated. }
$$

Thus $u_{x x}$ is lower order with respect to $v, w$ and, except for lower order terms, $v, w$ are solutions of the same differential equation as $u$. The same is true for the boundary conditions. Therefore we can estimate all second derivatives. Repeating the process, we can estimate any number of derivatives. 
We can now proceed in the same way as in [2], where we have considered first order systems, to obtain existence theorems for equations with variable coefficients. We approximate the differential equation by a stable difference approximation and prove, using summation by parts, that the corresponding estimates for the divided differences hold independently of the gridsize. In the limit of vanishing gridsize, we obtain the existence theorem. Since we can estimate any number of derivatives, it is well known, using Sobolev's theorem, that we can obtain similar, although local in time, estimates for quasilinear systems. By the same iterative methods as for first order symmetric hyperbolic systems, it follows that well-posedness extends locally in time to the quasilinear case, as well as other standard results such as the principle of finite speed of propagation.

\section{Appendix 2. Geometric Derivation of the Estimates}

We now use a geometric approach to show how the estimates established in Sec. 2 can be extended to the general initial-boundary value problem for the second order wave equation on a curved spacetime. A similar geometric approach has been used to implement the second order harmonic formulation of Einstein's equations as an evolution-boundary code based upon summation by parts [16]. See also [17] for another application of the geometric approach to the treatment of boundaries.

Using standard notation of general relativity, we consider the wave equation

$$
g^{a b} \nabla_{a} \nabla_{b} \phi=F
$$

for a massless scalar field propagating on a Lorentzian manifold with boundary of the form $M=[0, T] \times \Sigma$, where $\Sigma$ is a compact, 3-dimensional manifold with smooth boundary $\partial \Sigma$, each time-slice $\Sigma_{t}=\{t\} \times \Sigma$ is spacelike and the boundary $\mathcal{T}=[0, T] \times \partial \Sigma$ is timelike. Here $\nabla$ denotes the covariant derivative associated with the spacetime metric

$g$ with signature $(-,+,+,+)$. The initial-boundary value problem consists in finding solutions of (21) subject to the initial conditions

$$
\left.\phi\right|_{\Sigma_{0}}=f,\left.\quad n^{b} \nabla_{b} \phi\right|_{\Sigma_{0}}=h
$$

with Cauchy data $f$ and $h$ on $\Sigma_{0}$, and the boundary condition

$$
\left[\left(T^{b}+a N^{b}\right) \nabla_{b} \phi\right]_{\mathcal{T}}=q
$$


with data $q$ on $\mathcal{T}$. Here $n^{b}$ and $N^{b}$ denote the future-directed unit vector field to the timeslices $\Sigma_{t}$ and the outward unit normal vector field to $\mathcal{T}$, respectively; $T^{b}$ is an arbitrary future-directed timelike vector field which is tangent to the boundary surface $\mathcal{T}$; and $a>0$. The motion of the boundary is described geometrically by the hyperbolic angle $N^{b} n_{b}$.

Without loss of generality, we can assume that $T^{b}$ is normalized such that $g_{b c} T^{b} T^{c}=$ -1. A Sommerfeld boundary condition then corresponds to the choice $a=1$ for which $T^{b}+$ $N^{b}$ points in an outgoing characteristic (null) direction picked out by the geometry. See [16] for a numerical study of the harmonic Einstein system carried out with such a Sommerfeld condition, with evolution in the $T^{b}$ direction. In [16], the choice of matrices governing the tangential derivatives in the boundary conditions (13) was made for mathematical simplicity. In future work, we will explore more physically and geometrically motivated choices.

In order to establish estimates, we introduce the notation $\phi_{a}=\nabla_{a} \phi$ and the energy momentum tensor of the scalar field

$$
\Theta_{b}^{a}=\phi_{b} \phi^{a}-\frac{1}{2} \delta_{b}^{a} \phi^{c} \phi_{c}
$$

The essential idea is the use of an energy associated with a timelike vector $u^{a}=T^{a}+\delta N^{a}$, where $\delta>0$, so that $u^{a}$ points outward from the boundary. The corresponding energy $E(t)$ and the energy flux $\mathcal{F}(t)$ through the boundary $\Sigma_{t}$ are

$$
E(t)=\int_{\Sigma_{t}} u^{b} \Theta_{b}^{a} n_{a}
$$

and

$$
\mathcal{F}(t)=\int_{\partial \Sigma_{t}} u^{b} \Theta_{b}^{a} N_{a}
$$

It follows from the timelike property of $u^{a}$ that $E(t)$ is a norm for $\phi_{a}(t)$.

Energy conservation for the scalar field, i.e. integration by parts, gives

$$
\partial_{t} E=\mathcal{F}-\int_{\Sigma}\left(\Theta_{a b} \nabla^{a} u^{b}+u^{a} \phi_{a} F\right)
$$

so that

$$
\partial_{t} E \leq \mathcal{F}+\text { const. }\left(E+\int_{\Sigma} F^{2}\right) .
$$


The required estimates arise from considering the flux density

$$
\begin{aligned}
& u^{b} \Theta_{b}^{a} N_{a}=N^{a} \phi_{a} T^{b} \phi_{b}+\delta\left(N^{a} \phi_{a}\right)^{2}-\frac{\delta}{2} \phi^{a} \phi_{a} \\
& =N^{a} \phi_{a} T^{b} \phi_{b}+\frac{\delta}{2}\left(N^{a} \phi_{a}\right)^{2}+\frac{\delta}{2}\left(T^{a} \phi_{a}\right)^{2}-\frac{\delta}{2} H^{a b} \phi_{a} \phi_{b} \\
& =-\frac{\delta}{2}\left(\left(N^{a} \phi_{a}\right)^{2}+\left(T^{a} \phi_{a}\right)^{2}+H^{a b} \phi_{a} \phi_{b}\right)+N^{a} \phi_{a} T^{b} \phi_{b}+\delta\left(N^{a} \phi_{a}\right)^{2}+\delta\left(T^{a} \phi_{a}\right)^{2}
\end{aligned}
$$

where $H_{b c}=g_{b c}+T_{b} T_{c}-N_{b} N_{c}$ is the positive definite metric in the tangent space of the boundary orthogonal to $T^{a}$. By using the boundary condition to eliminate $T^{a} \phi_{a}$ in the last group of terms, we obtain

$$
\begin{array}{r}
u^{b} \Theta_{b}^{a} N_{a}=-\frac{\delta}{2}\left(\left(N^{a} \phi_{a}\right)^{2}+\left(T^{a} \phi_{a}\right)^{2}+H^{a b} \phi_{a} \phi_{b}\right) \\
+\left(-a+\delta\left(1+a^{2}\right)\right)\left(N^{a} \phi_{a}\right)^{2}+(1-2 a \delta) N^{a} \phi_{a} q+\delta q^{2}
\end{array}
$$

so that

$$
\begin{array}{r}
u^{b} \Theta_{b}^{a} N_{a}=-\frac{\delta}{2}\left(\left(N^{a} \phi_{a}\right)^{2}+\left(T^{a} \phi_{a}\right)^{2}+H^{a b} \phi_{a} \phi_{b}\right) \\
+\left(-a(1-\epsilon)+\delta\left(1+a^{2}\right)\right)\left(N^{a} \phi_{a}\right)^{2} \\
-\epsilon a\left(N^{a} \phi_{a}-\frac{(1-2 a \delta)}{2 a \epsilon} q\right)^{2}+\left(\delta+\frac{(1-2 a \delta)^{2}}{4 a \epsilon}\right) q^{2} .
\end{array}
$$

In the above equation, we have introduced the $\epsilon$-terms, with $0 \leq \epsilon \leq 1$, in order to establish the inequality

$$
\begin{aligned}
u^{b} \Theta_{b}^{a} N_{a} \leq- & \frac{\delta}{2}\left(\left(N^{a} \phi_{a}\right)^{2}+\left(T^{a} \phi_{a}\right)^{2}+H^{a b} \phi_{a} \phi_{b}\right) \\
+(-a(1-\epsilon) & \left.+\delta\left(1+a^{2}\right)\right)\left(N^{a} \phi_{a}\right)^{2} \\
+ & \left(\delta+\frac{(1-2 a \delta)^{2}}{4 a \epsilon}\right) q^{2} .
\end{aligned}
$$

The boundary estimate of $\phi_{a}$ now follows by requiring $-a(1-\epsilon)+\delta\left(1+a^{2}\right) \leq 0$, which guarantees that $u^{a}$ is timelike. With the choice

$$
\delta=\frac{a(1-\epsilon)}{\left(1+a^{2}\right)},
$$

(22) and (23) give

$$
\begin{array}{r}
\partial_{t} E+\int_{\partial \Sigma} \frac{\delta}{2}\left(\left(N^{a} \phi_{a}\right)^{2}+\left(T^{a} \phi_{a}\right)^{2}+H^{a b} \phi_{a} \phi_{b}\right) \\
\leq \text { const. }\left(E+\int_{\Sigma} F^{2}+\int_{\partial \Sigma} q^{2}\right) .
\end{array}
$$


In analogy with (19), this leads to the required estimate of the gradient $\phi_{a}$ on the boundary (as well as the usual estimate of the gradient at a fixed time) to prove that the problem is strongly well-posed. An estimate of $\phi$ itself follows by introducing a mass term in (21) through the change of variable $\phi \rightarrow e^{\gamma t} \phi^{\prime}$, as described in Appendix 1. Energy estimates for the problem $\left(1^{\prime}\right),(2),(3)$ in Sec. 2 follow from the choice $T^{b}=\left(T^{t}, T^{x}, T^{y}, T^{z}\right)=$ $\tau\left(\alpha^{\prime}, 0,-\beta_{1}^{\prime},-\beta_{2}^{\prime}\right)$ with $N^{b}=-\nu g^{b a} \nabla_{a} x=-\nu(c, 1,0,0)$, where $\tau$ and $\nu$ are positive normalization constants and $\alpha^{\prime}$ and $\beta_{i}^{\prime}$ are related to the coefficients $\alpha$ and $\beta_{i}$ in (2) by

$$
\begin{array}{r}
\alpha=\frac{\tau \alpha^{\prime}}{a \nu}-c \\
\beta_{i}=\frac{\tau \beta_{i}^{\prime}}{a \nu}, \quad i=1,2 .
\end{array}
$$


Acknowledgments

The work of O. R. was supported in part by CONICET, SECYT-UNC and NSF Grant INT0204937 to Louisiana State University. The work of O. S. was supported in part by grant CIC 4.20 to Universidad Michoacana. The work of J. W. was supported by NSF grant PH-0553597 to the University of Pittsburgh.

\section{References}

[1] H.-O. Kreiss and J. Winicour, Class. Quantum Grav. 23, 405-420, (2006).

[2] H.-O. Kreiss and J. Lorenz, Initial-Boundary Value Problems and the NavierStokes Equations, 1989, Reprint SIAM CLASSICS, 2004.

[3] B. Gustafsson, H.-O. Kreiss and J. Oliger, Time dependent problems and difference methods, (A Wiley-Interscience Publication, 1995).

[4] H.-O. Kreiss, N. A. Petersson, SIAM J. Sci. Comput. 27, 1141 (2006).

[5] H.-O. Kreiss, N. A. Petersson, and J. Yström, SIAM J. Numer. Anal. 42, 1292 (2004).

[6] M. C. Babiuc, B. Szilágyi and J. Winicour, Phys.Rev. D73, 064017 (2006).

[7] O. Sarbach, Absorbing boundary conditions for Einstein's field equations, arXiv:0708.4266 [gr-qc]

[8] H. Friedrich and G. Nagy, Commun. Math. Phys. 201, 619 (1999).

[9] Y. Foures-Bruhat, Acta Math. 88, 141 (1952).

[10] H. Friedrich, Class. Quantum Grav. 13, 1451 (1996).

[11] F. Pretorius, Phys. Rev. Lett. 95, 121101 (2005).

[12] F. Pretorius, Class. Quantum Grav. 23, S529 (2006).

[13] L. Lindblom, M. Scheel, L. E. Kidder, R. Owen and O. Rinne, Class. Quantum Grav. 23, S447 (2006).

[14] C. Palenzuela, I. Olabarrieta, L. Lehner and S. Liebling, Phys.Rev. D75, 064005 (2007).

[15] B. Szil' agyi, D. Pollney, L. Rezzolla, J. Thornburg and J. Winicour, Class. Quantum Grav. 24, S275 (2007).

[16] M. C. Babiuc, H.-O. Kreiss and J. Winicour, Phys. Rev. D75, 044002 (2007). 
[17] G. Calabrese, L. Lehner, D. Neilsen, J. Pullin, O. Reula, O. Sarbach, and M. Tiglio, Class. Quantum Grav. 20, L245 (2003). 